\title{
Ionizing radiation promotes advanced malignant traits in nasopharyngeal carcinoma via activation of epithelial-mesenchymal transition and the cancer stem cell phenotype
}

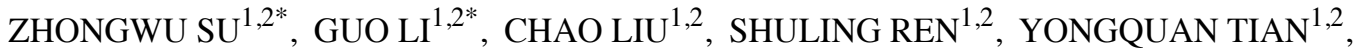 \\ YONG LIU ${ }^{1,2}$ and YUANZHENG QIU ${ }^{1,2}$ \\ ${ }^{1}$ Department of Otolaryngology Head and Neck Surgery, Xiangya Hospital, Central South University, Changsha, \\ Hunan 410008; ${ }^{2}$ Otolaryngology Major Disease Research Key Laboratory of Hunan Province, \\ Changsha, Hunan 410008, P.R. China
}

Received December 10, 2015; Accepted January 11, 2016

DOI: $10.3892 / o r .2016 .4768$

\begin{abstract}
Post-irradiation residual mass and recurrence always suggest a worse prognosis for nasopharyngeal carcinoma (NPC). Our study aimed to investigate the malignant behaviors of post-irradiation residual NPC cells, to identify the potential underlying mechanisms and to search for appropriate bio-targets to overcome this malignancy. Two NPC cell lines were firstly exposed to $60 \mathrm{~Gy}$ irradiation, and residual cells were collected. In our previous study, colony formation assay detected the radioresistance of these cells. Here, the CCK-8 assay examined the cell sensitivity to paclitaxel and cisplatin. Wound-healing and Transwell assays were performed to investigate cell motility and invasion capabilities. Inverted phase-contrast microscopy was used to observe and photograph the morphology of cells. Expression levels of epithelial-mesenchymal transition (EMT)-related proteins were detected by western blot assay in NPC cells and tissues. The mRNA levels of cancer stem cell (CSC)-related genes were detected via qRT-PCR. The results revealed that residual NPC cells exhibited enhanced radioresistance and cross-resistance to paclitaxel and cisplatin. Higher capacities of invasion and migration were also observed. An elongated morphology with pseudopodia formation and broadening in the intercellular
\end{abstract}

Correspondence to: Dr Yong Liu or Dr Yuanzheng Qiu, Department of Otolaryngology Head and Neck Surgery, Xiangya Hospital, Central South University, 87 Xiangya Road, Changsha, Hunan 410008, P.R. China

E-mail: braver19840112@hotmail.com

E-mail: xyqyz@hotmail.com

${ }^{*}$ Contributed equally

Key words: epithelial-mesenchymal transition, nasopharyngeal carcinoma, radiotherapy, chemoresistance, metastasis, cancer stem cell space was observed in the residual cells. Downregulation of E-cadherin and upregulation of vimentin were detected in the residual NPC cells and tissues. CSC-related Lgr5 and c-myc were significantly upregulated in the CNE-2-Rs and 6-10B-Rs radioresistance cells. Higher proportions of Lgr5+ cells were observed in radioresistant cells via immunofluorescent staining and flow cytometry. In conclusion, our study demonstrated that residual NPC cells had an advanced malignant transition and presented with both EMT and a CSC phenotype. This provides a possible clue and treatment strategy for advanced and residual NPC.

\section{Introduction}

Nasopharyngeal carcinoma (NPC) is one of the most common head and neck malignancies in Southeast Asia and Southern China (1). Radiotherapy is a routine treatment for NPC patients (2). With more accurate intensity-modulated radiation therapy and adjuvant chemotherapy, the 5-year survival of NPC patients has reached more than $60 \%$ (3). Clinically, posttreatment recurrence and residual mass are still obstacles to successful treatment in a small population of NPC cases. NPC patients with residue are always considered to be refractory to salvage irradiation and chemotherapy and present with higher local or distant metastasis, resulting in a worse prognosis (4). However, the underlying mechanisms and malignant behaviors of residual NPC remains unclear.

Several situations restrict the study of these patients. In routine clinical practice, recurrence and residual NPC tissues are difficult to obtain for the reason that surgery is not the first line therapeutic choice for these NPC patients. Meanwhile, the residues are usually located in the deeper para-pharyngeal space covered with fibrotic scar and necrotic tissue after full course radiotherapy, which also restrict successful biopsy. Thus, we established post-irradiation residual NPC cells by exposure to irradiation in our previous study, which, to some extent, imitate the patients with a residual mass after a full course of radiotherapy $(5,6)$. Using these cells, we detected 
their malignant alteration in regards to radioresistance, chemoresistance, motility and invasion capabilities.

Epithelial-mesenchymal transition (EMT) is a process by which cells change their original epithelial morphology and are dispersed with the disappearance of inter-cellular connections, and change into long fibroblast-like cells (7). It is well known that EMT gifts tumor cells with more powerful metastatic potency (7). Recently, compelling evidence indicates that EMT transition in tumor cells also contributes to other malignant behaviors, such as chemoresistance and radioresistance $(8,9)$. On the other hand, cancer stem cells (CSCs) are also considered as another crucial mechanism for the tumor to survive in extreme environments, such as irradiation (10) or chemotherapeutic agents (11). These intra-tumoral cells are generated and are maintained in a small proportion, but have the ability to self-renew and to differentiate in multidirections which include EMT (10). Thus, we simultaneously focused on cellular morphological changes, detected EMT and CSC biomarker levels, and aimed to identify the underlying mechanisms to reverse the occurrence and residues in NPC.

\section{Materials and methods}

Cell culture. The poorly differentiated NPC cell lines CNE-2 and 6-10B were provided by the Cell Center of Central South University (Changsha, China). The radioresistant NPC cells derived from the CNE-2 and 6-10B cells were established as previously described (5) and were termed CNE-2-Rs and 6-10B-Rs, respectively. Cells were cultured in RPMI-1640 medium (Hyclone, Waltham, MA, USA) supplemented with $10 \%$ fetal bovine serum (FBS) and $1 \%$ penicillin and streptomycin (all from Gibco, MA, USA). Cell cultures were maintained in a humidified atmosphere of $5 \% \mathrm{CO}_{2}$ at $37^{\circ} \mathrm{C}$. Cell morphology was monitored with an inverted phasecontrast microscope (Leica, Wetzlar, Germany).

Patients and tissue preparation. Three coupled NPC tissues before/post-radiotherapy were obtained from October 2011 to October 2014 at the Department of Otolaryngology Head and Neck Surgery, Xiangya Hospital of Central South University (Changsha, China). None of the patients had a previous malignancy and did not undergo chemo/radiotherapy. All specimens were snap-frozen immediately and stored in liquid nitrogen for further protein extraction. The present study was approved by the Ethics Committee of Xiangya Hospital of Central South University. Prior patient consent was obtained from all the patients.

Cell viability assay. Cell viability was assessed by the CCK-8 kit (Beyotime, Shanghai, China) as described previously (12). Briefly, the cells were seeded in 96-well plates at $5,000 /$ well and allowed to adhere to the plate overnight. The cells were then exposed to different concentrations of paclitaxel or cisplatin (both from Sigma, USA) for another $48 \mathrm{~h}$. Absorbance values were expressed as percentages relative to the controls. Resistant index was calculated by $\mathrm{IC}_{50}$ (resistant cells) $/ \mathrm{IC}_{50}$ (parental cells) where $\mathrm{IC}_{50}$ is the half maximal inhibitory concentration. Each experiment was performed in triplicate.
Wound-healing assay. Wound-healing assay was performed as previously described (13). The cells were seeded in 6-well plates at $2 \times 10^{5} /$ well and allowed to grow to almost confluency. The cells were washed twice with PBS and incubated in serum-free medium for another $24 \mathrm{~h}$. The cell monolayer was wounded with a sterile $10-\mu 1$ pipette tip. Then the cells were cultured in medium supplemented with $2 \%$ FBS for another $48 \mathrm{~h}$. Cell migration was calculated by measuring the distance covered by migrating cells and further divided by that of the original wound. The experiment was carried out in triplicate.

Invasion assay. The invasion assay was also described previously (13). In brief, chambers coated with Matrigel (BD Biosciences, Bedford, MA, USA) were incubated at $37^{\circ} \mathrm{C}$ for $30 \mathrm{~min}$. The cells were seeded in the upper well and incubated for 24 or $48 \mathrm{~h}$. The cells in the upper well were removed and the cells that had invaded to the lower side of the filter were fixed with methanol and stained with crystal violet. The number of invading cells was quantified by counting in 5 random fields (x200 field). This experiment was performed in triplicate.

Western blotting. Western blot assay was performed as previously described (12). In brief, the total protein was extracted and separated by SDS-PAGE. Then the separated proteins were transferred to PVDF membranes. The membranes were incubated with the corresponding primary antibodies followed by the relevant secondary antibody. Primary antibodies used in the present study were monoclonal rabbit anti-E-cadherin (1:1,000), anti-vimentin (1:500) (both from Cell Signalling Technology, Danvers, MA, USA) and monoclonal mouse anti- $\beta$-actin (1:1,000; Beyotime).

Quantitative real-time reverse transcription-PCR ( $q R T-P C R)$. Briefly, cDNA was synthesized from total RNA using a PrimeScript RT reagent kit with a DNA Eraser (Takara, Shiga, Japan). Primers for genes related to cancer stem cells were designed and synthesized. Then, qPCR assays were performed using a Bio-Rad IQ5 ${ }^{\text {TM }}$ Multicolor Real-Time qRT-PCR detection system (Bio-Rad, Hercules, CA, USA). The mRNA expression levels were detected as previously described (5) using specific primer sequences (Table I). The expression levels were measured in terms of the cycle threshold $(\mathrm{Ct})$ and were then normalized to GAPDH expression using the $2^{-\Delta \Delta \mathrm{Ct}}$ method $(5,14)$.

Immunofluorescent staining and flow cytometry assay. The cells were washed with phosphate-buffered saline (PBS, Hyclone, Waltham, MA, USA) and blocked with $0.05 \%$ bovine serum albumin (Beyotime, Shanghai, China) in PBS. The cells were diluted to $10^{\wedge} 6-10^{\wedge} 7 / \mathrm{ml}$ and stained with rabbit antiLGR5-FITC (Bioss, Beijing, China; cat. no: bs-1117R-FITC, 1:100 dilution) at $4^{\circ} \mathrm{C}$ for $1 \mathrm{~h}$. After being washed with PBS, the cells were used for a FACScalibur through a flow cytometer (Becton Dickinson, San Jose, CA, USA) and analyzed using WinMDI software.

Statistical analysis. The statistical analyses were performed using SPSS 17.0 software. The quantitative data are presented as the mean \pm standard deviation (SD). Statistical comparisons between two groups were performed using the Student's t-test. In all cases, $\mathrm{p}<0.05$ was considered statistically significant. 
A

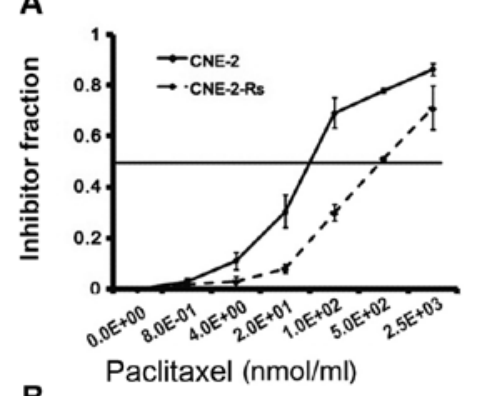

B

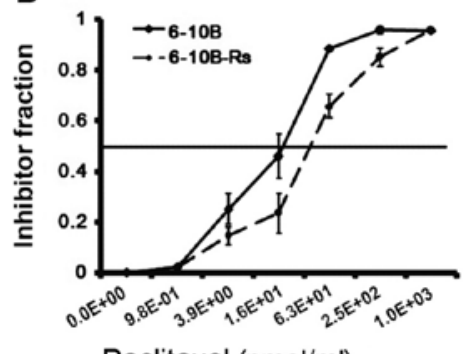

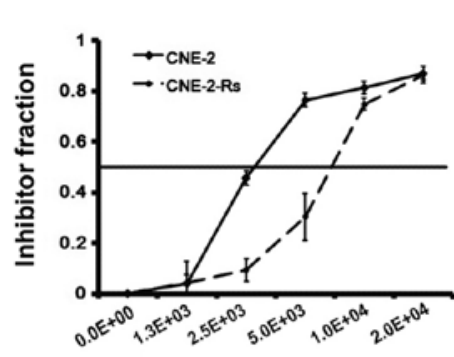

Cisplatin $(\mathrm{nmol} / \mathrm{ml})$

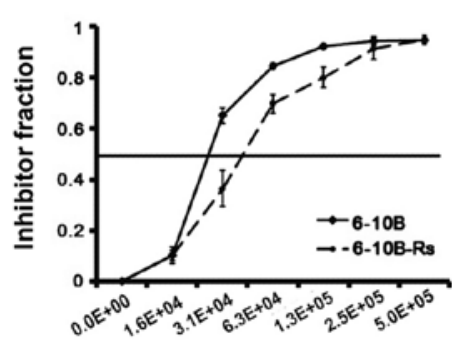

Cisplatin $(\mathrm{nmol} / \mathrm{ml})$
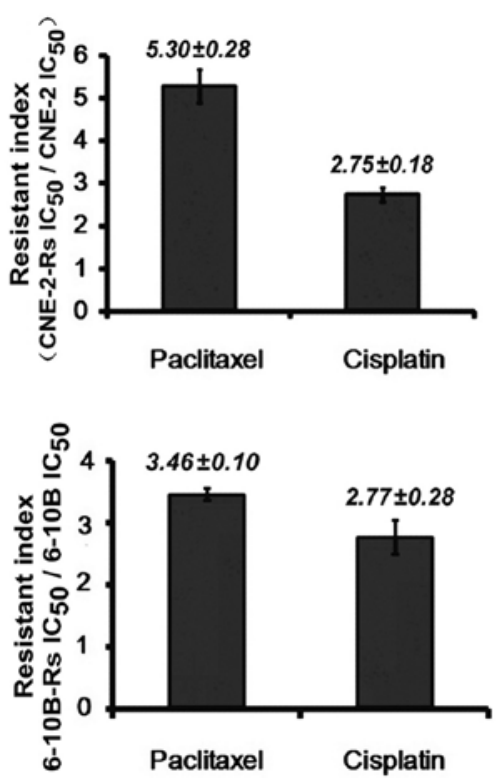

Figure 1. Irradiation induces increasing chemoresistance in the NPC cells. (A) The $\mathrm{IC}_{50}$ values for paclitaxel in the CNE-2-Rs and CNE-2 cells were $38.2 \pm 6.09$ and $7.24 \pm 1.29 \mathrm{nmol} / \mathrm{ml}$. The $\mathrm{IC}_{50}$ values for cisplatin in the CNE-2-Rs and CNE-2 cells were 8.32 \pm 0.83 and $3.02 \pm 0.34\left(\mathrm{x} 10^{4}\right) \mathrm{nmol} / \mathrm{ml}$. The calculated resistant indices of paclitaxel and cisplatin were $5.28 \pm 0.39$ and $2.75 \pm 0.18(\mathrm{p}<0.05)$, respectively. (B) The IC ${ }_{50}$ values for paclitaxel in the 6-10B-Rs and 6-10B cells were $60.12 \pm 3.71$ and $17.38 \pm 0.59 \mathrm{nmol} / \mathrm{ml}$. The $\mathrm{IC}_{50}$ values for cisplatin in the 6-10B-Rs and 6-10B cells were $4.68 \pm 0.37$ and $1.67 \pm 0.24\left(\mathrm{x} 10^{4}\right) \mathrm{nmol} / \mathrm{ml}$. The resistant indices of paclitaxel and cisplatin were $3.46 \pm 0.10$ and $2.77 \pm 0.28(\mathrm{p}<0.05)$, respectively.

Table I. Gene primers.

Gene Primer sequence

\section{Lgr5}

F: 5'-CTCTTCCTCAAACCGTCTGC-3' R: 5'-GATCGGAGGCTAAGCAACTG-3'

c-myc F: 5'-GTCAGTATCACGCCCGTTTT-3' R: 5'-GCTTCCTTTACGCACTTGGT-3'

CD117 F: 5'-GCACAGCCTTGAGCCTACTC-3' R: 5'-TACGAATGCATGGGCAGTAA-3'

Bmi 1 F: 5'-CCAGGGCTTTTCAAAAATGA-3' ALDH1 R: 5'-CCGATCCAATCTGTTCTGGT-3' CD133 F: 5'-AAGCCAAGTGCTCTATCA-3' R: 5'-TCAACATCCTCCTTATCTC-3'

CD133 F: 5'-TTGTGGCAAATCACCAGGTA-3' R: 5'-TCAGATCTGTGAACGCCTTG-3' PD-L1 F: 5'-TATGGTGGTGCCGACTACAA-3' R: 5'-TGCTTGTCCAGATGACTTCG-3' CD24 F: 5'-GCCAGTCTCTTCGTGGTCTC-3' R: 5'-CCTGTTTTTCCTTGCCACAT-3' GAPDH

$$
\text { F: 5'-TCCAAAATCAAGTGGGGCGA-3' }
$$$$
\text { R: 5'-AGTAGAGGCAGGGATGATGT-3' }
$$

F, forward; R, reverse.

\section{Results}

Irradiation induces chemoresistance in NPC cells. To investigate the potential impact of irradiation on chemosensitivity,
NPC radioresistant cells CNE-2-Rs and 6-10B-Rs, established via exposure to gradually increasing doses of irradiation in our previous study (5), were used in the following experiments. We initially applied the CCK-8 assay to evaluate the sensitivity of radioresistant NPC cells to routine chemoagents including paclitaxel and cisplatin. Our results revealed that both the $\mathrm{IC}_{50}$ values of paclitaxel and cisplatin in the radioresistant NPC CNE-2-Rs were increased 5.28$(38.2 \pm 6.09$ vs. $7.24 \pm 1.29 \mathrm{nmol} / \mathrm{ml})$ and 2.75 -fold $(8.32 \pm 0.83$ vs. $3.02 \pm 0.34 \mathrm{nmol} / \mathrm{ml})$, compared with the parental CNE-2 cells (Fig. 1A). Meanwhile, similar data were obtained in the radioresistant 6-10B-Rs cells, which showed 3.46$(60.12 \pm 3.71$ vs. $17.38 \pm 0.59 \mathrm{nmol} / \mathrm{ml})$ and 2.77 -fold $(4.68 \pm 0.37$ vs. $1.67 \pm 0.24 \mathrm{nmol} / \mathrm{ml})$ more resistance to paclitaxel and cisplatin (Fig. 1B). Collectively, these results indicate that the radioresistant NPC cells acquired resistance to paclitaxel and cisplatin after surviving from exposure to irradiation.

Irradiation promotes the migration of the NPC cells. The migratory capacity of the radioresistant NPC cells was then investigated by wound healing assay. As indicated in Fig. 2, the migration of NPC cells was observed and photographed at 0 and $48 \mathrm{~h}$, respectively. Our data demonstrated that the migration rate was obviously increased in the radioresistant NPC cells compared to the parental NPC cells (CNE-2-Rs cells: $58.04 \pm 7.22 \%$ vs. CNE-2 cells: $38.23 \pm 6.15 \%, \mathrm{p}<0.05$; 6-10B-Rs cells: $87.09 \pm 10.72 \%$ vs. $6-10$ B cells $44.71 \pm 3.98 \%$, $\mathrm{p}<0.05)$. These results indicate that irradiation can promote the migration of NPC cells in vitro.

Irradiation enhances the invasion of the NPC cells. The invasive capacity of the radioresistant NPC cells was also examined by Transwell assay. As indicated in Fig. 3A, the numbers of 
A

CNE-2

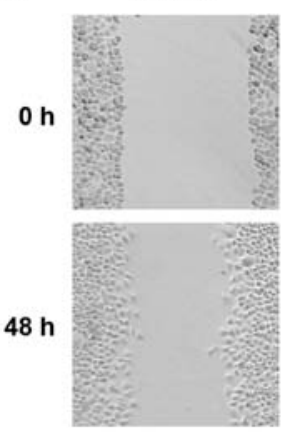

B

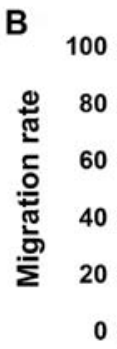

CNE-2-Rs
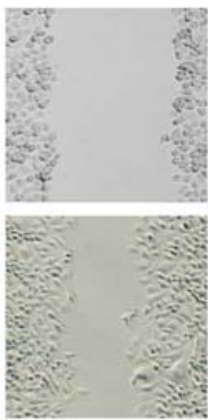

$\mathrm{Oh}$

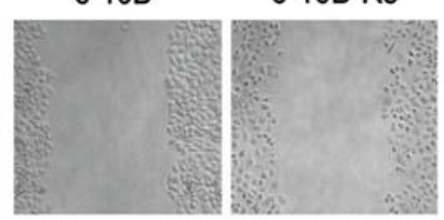

$48 \mathrm{~h}$

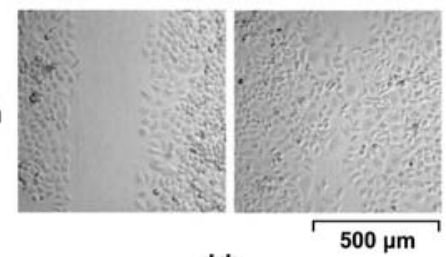

$\star *$

$500 \mu \mathrm{m}$
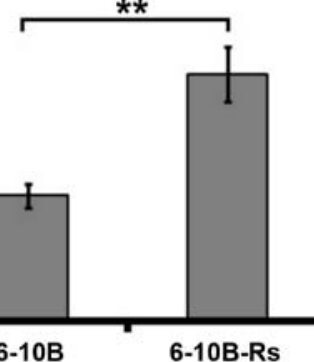

Figure 2. Irradiation promotes the migration of NPC cells. (A and B) The migration of the NPC cells was observed and photographed at 0 and $48 \mathrm{~h}$ (upper, $\mathrm{x} 100$ field). The calculated migration rates were $58.04 \pm 7.22$ and $38.23 \pm 6.15 \%(\mathrm{p}<0.05)$ in the CNE-2-Rs and CNE-2 cells, respectively, and the calculated migration rates were $87.09 \pm 10.72$ and $44.71 \pm 3.98 \%(\mathrm{p}<0.01)$ in the 6-10B-Rs and 6-10B cells, respectively (lower, $\mathrm{x} 100$ field).

A
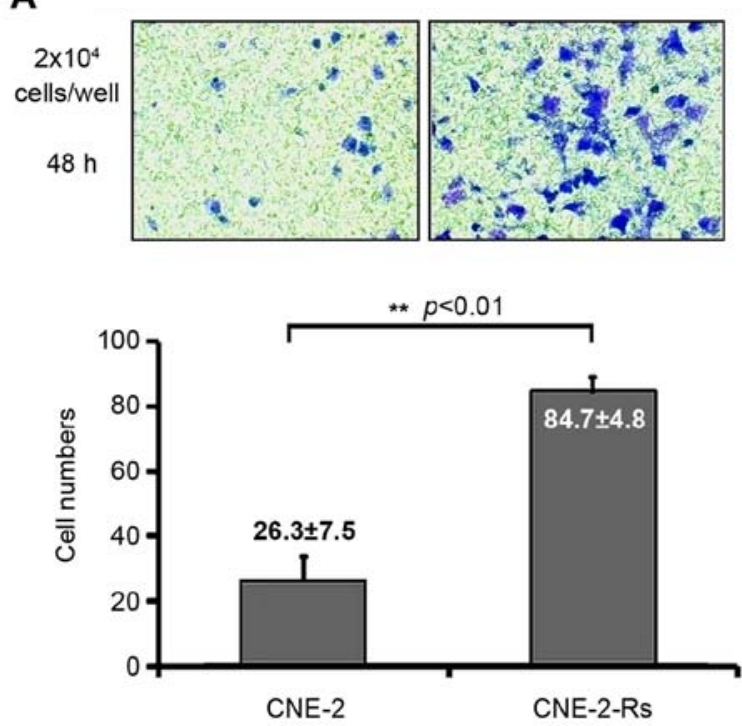

B
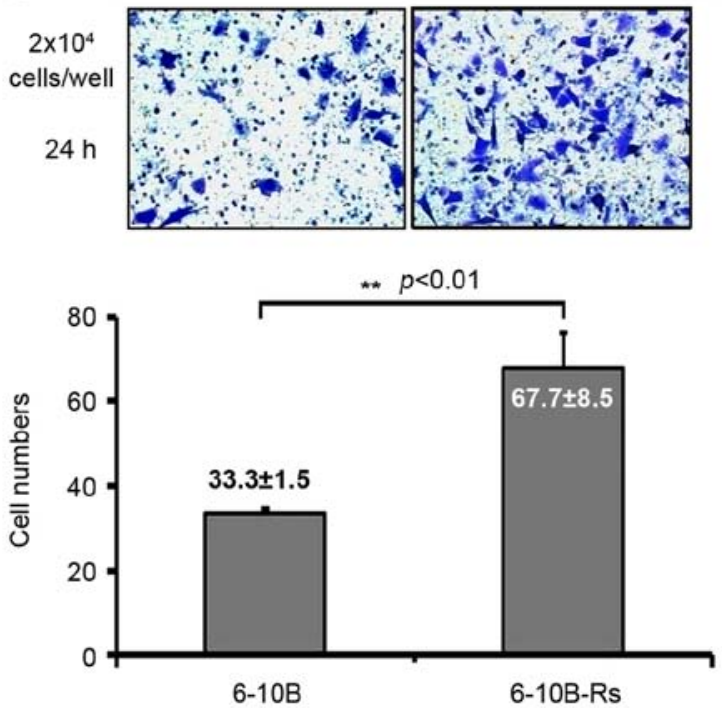

Figure 3. Irradiation promotes the invasion of NPC cells. (A) The number of invading CNE-2 and CNE-2-Rs cells after 48 h were $26.3 \pm 7.5$ and $84.7 \pm 4.8$ ( $\mathrm{p}<0.01$ ), respectively. (B) The number of invading 6-10B and 6-10B-Rs cells after $24 \mathrm{~h}$ were $33.3 \pm 1.5$ and $67.7 \pm 8.5$ ( $\mathrm{p}<0.01$ ).

invading CNE-2 and CNE-2-Rs cells on the bottom sides of the Matrigel-coated membrane after $48 \mathrm{~h}$ were $26.3 \pm 7.5$ and $84.7 \pm 4.8(\mathrm{p}<0.01)$, respectively. Similarly, the numbers of invading 6-10B and 6-10B-Rs cells after $24 \mathrm{~h}$ were $33.3 \pm 1.5$ and $67.7 \pm 8.5$ ( $p<0.01)$, respectively (Fig. 3B). Taken together, the above results suggest that irradiation can enhance the metastatic phenotype including migration and invasion in the NPC cells.

Irradiation leads to EMT change in the NPC cells. Our above results revealed that irradiation induces chemoresistance to paclitaxel and cisplatin, and promotes migration and invasion of NPC cells in vitro. However, the underlying mechanisms that promote radioresistance, chemoresistance and metastasis in NPC remain to be clarified. In the phase of establishing radioresistant NPC cells, we observed significant morphological changes in the radioresistant NPC cells. Under phase-contrast microscopy, radioresistant CNE-2-Rs and 6-10B-Rs cells were found to become elongated with pseudopodia formation, a decrease in cell-to-cell contact, similar to the shape of fibroblasts (Fig. 4A). These morphological changes were consistent 


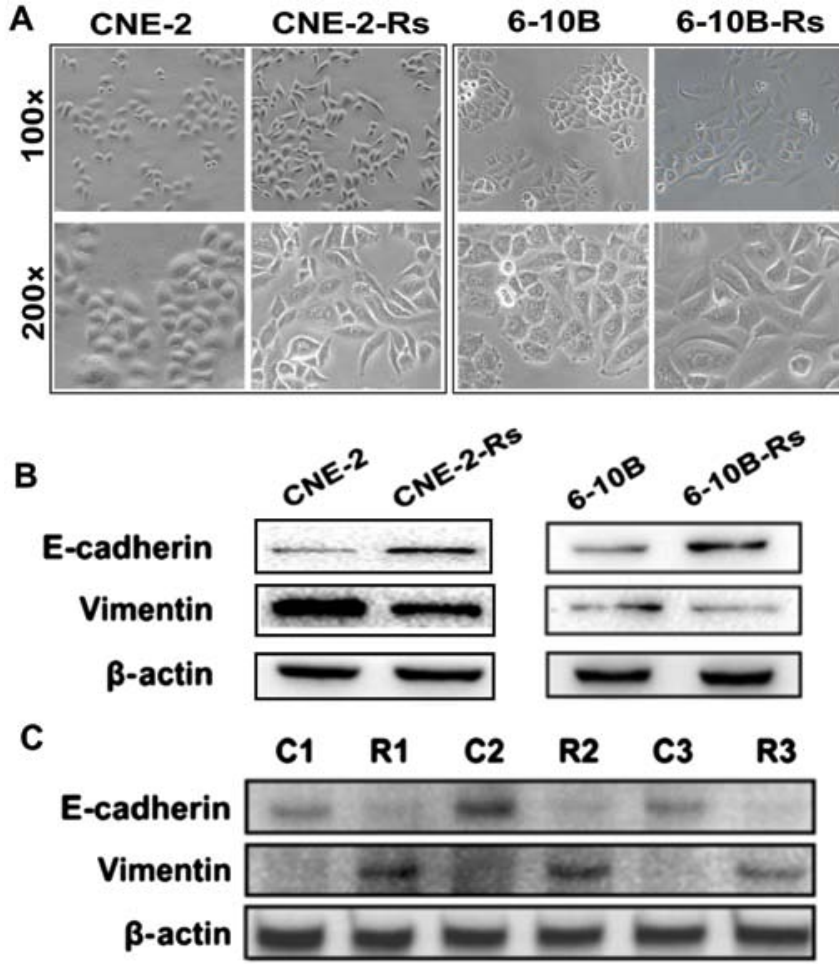

Figure 4. Irradiation induces the EMT phenotype in NPC cells. (A) The morphology of NPC cells was observed and photographed (upper, $\mathrm{x} 100$ field; lower, $\mathrm{x} 200$ field). The parental CNE-2 and 6-10B cells showed a pebble-like phenotype. The radioresistant CNE-2-Rs and 6-10B cells became elongated with pseudopodia formation, and loose in cell-to-cell contact. (B) Western blot assays showed that the expression of E-cadherin was decreased, while expression of vimentin was increased, in the CNE-2-Rs and 6-10B-Rs cells compared with their parental cells. (C) In three coupled NPC tissues, less E-cadherin expression and more vimentin expression were detected in residual NPC specimens (R1-R3) than in the corresponding tissues (C1-C3) before radiotherapy.

with canonical EMT formation, which has been reported to tightly correlate with radioresistance, chemoresistance and metastasis (15). Therefore, the molecules associated with EMT were further examined. Our data clearly indicated that CNE-2-Rs and 6-10B-Rs cells displayed higher expression of mesenchymal marker vimentin and lower expression of epithelial protein E-cadherin (Fig. 4B). These findings were further validated in clinical NPC samples that experienced irradiation. Three residual NPC specimens post-radiotherapy were obtained. Western blot analysis detected decreased expression of E-cadherin and elevated expression of vimentin in the NPC residues, compared with the NPC samples before radiotherapy (Fig. 4C). These results suggest that irradiation promotes the emergence of EMT, which may be involved in the observed chemoresistance and metastasis in NPC.

Irradiation increases molecules associated with cancer stem cells (CSCs). CSCs persist in tumors as a distinct population and cause cancer progression and relapse by giving rise to new tumors (16). CSCs are involved in diverse cancer malignant behaviors including metastasis, radioresistance and chemoresistance (16). Therefore, we hypothesized that irradiation may also increase the number of CSCs and promote chemoresistance and metastasis. To test this hypothesis, qRT-PCR assays were used to evaluate molecular markers associated with

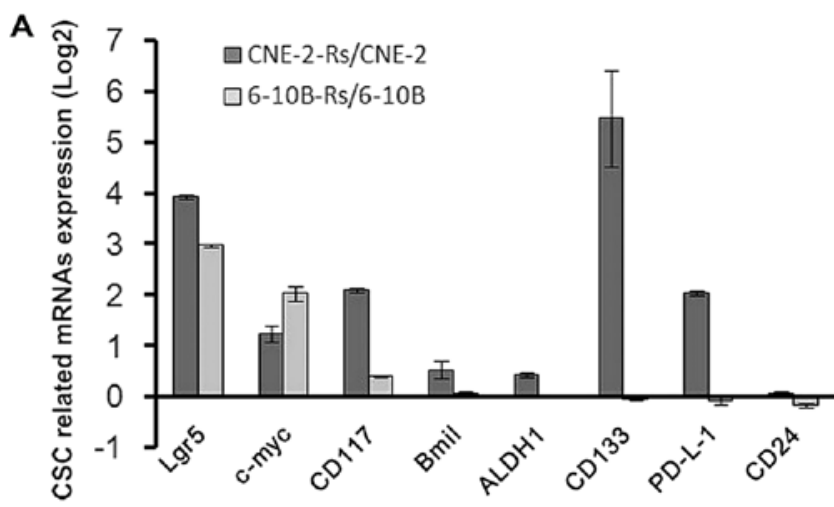

B

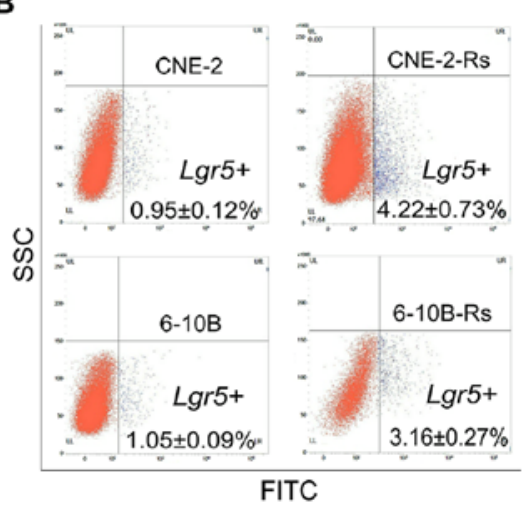

Figure 5. Irradiation induces cancer stem cell (CSC) molecular alterations in the NPC cells. (A) CSC markers Lgr5 and c-myc were upregulated in the CNE-2-Rs and 6-10B-Rs radioresistant cells. (B) B. Higher proportions of Lgr5+ cells were observed in radioresistant cells via immunofluorescent staining and flow cytometry. The Lgr5+ proportion was $4.22 \pm 0.73 \%$ and $0.95 \pm 0.12 \%(\mathrm{p}<0.05)$ in the CNE-2-Rs and CNE- 2 cells, respectively, and the Lgr5+ proportion was $3.16 \pm 0.27 \%$ and $1.05 \pm 0.09 \%(\mathrm{p}<0.05)$ in the $6-10 \mathrm{~B}-\mathrm{Rs}$ and $6-10 \mathrm{~B}$ cells, respectively.

CSCs. Our results showed that Lgr5 and c-myc had consistent changes (fold-change $>2$ ) in the CNE-2-Rs and 6-10B-Rs cells, when compared with the parental cells (Fig. 5A). Then, Lgr5+ proportions of the cells were detected by immunofluorescent staining and flow cytometry. The Lgr5+ proportion was $4.22 \pm 0.73 \%$ and $0.95 \pm 0.12 \%(\mathrm{p}<0.05)$ in the CNE-2-Rs and CNE-2 cells, respectively, and the Lgr5+ proportion was $3.16 \pm 0.27 \%$ and $1.05 \pm 0.09 \%(\mathrm{p}<0.05)$ in the $6-10 \mathrm{~B}-\mathrm{Rs}$ and 6-10B cells, respectively (Fig. 5B). The data suggest that irradiation may induce chemoresistance and metastasis via increasing CSCs, which is indicated by increased expression of specific CSC markers.

\section{Discussion}

Clinically, the difficulty in obtaining specimens is still an obstacle for the study of NPC post-irradiation residue and recurrence. In order to better understand the underlying mechanism, the establishment of post-irradiation residual NPC cells is the main choice for many laboratories and research teams. Three methods have been widely accepted to obtain post-irradiation NPC cell lines, including low-dose repeated irradiation (17), sublethal dose irradiation (18) and gradient increasing irradiation (6). Compared with the first 
two patterns, accumulating doses in a gradient irradiation pattern of up to 60-70 Gy most closely resembles the clinical doses for NPC patients. Therefore, our group chose a gradient increasing irradiation pattern and successfully established two post-irradiation residual NPC cell lines $(5,6)$.

In some cases, post-irradiation residual cells were found to be also accompanied with advanced radioresistance, and were considered to be radioresistant cells $(6,17)$. Similarly, two residual NPC cell lines screened in our previous research were named radioresistant CNE-2-Rs and 6-10B-Rs cells, respectively (6). Previous research has only focused on the mechanism of radioresistance rather than other malignant behaviors. Our results showed chemoresistance as well as aggressive motility and invasion capability in residual NPC cells. These findings, to some extent, may be the answer for treatment failure and the poorer prognosis of NPC patients with post-irradiation residue and recurrence.

Notably, following the total irradiation dose of the gradually accumulation, NPC cells transformed from epithelial cells into a mesenchymal phenotype. Concurrently, a loss of epithelial marker E-cadherin and an increase in the mesenchymal molecule vimentin were also observed in the post-irradiation cells. Thus, we considered that the NPC cells went through an EMT process, which partially explains the higher invasion and metastasis observed in the post-irradiation cells. In addition, in three pairs of patient tissues from pre- and postirradiation, EMT markers exhibited the same alterations as in the residual cells, further supporting that irradiation may induce EMT in NPC. Several reports suggested the potential connections between the EMT phenotype and post-irradiation residues, in malignancies of the stomach (19), prostate (20) and cervical cancer (21). The mechanisms involved included activation of the WNT/ $\beta$-catenin (22), PI3K/Akt/mTOR (23), and $N F-\kappa B$ pathways (24). Inspiringly, our group firstly found this connection in NPC cells, and showed that cells with an EMT phenotype not only had a greater radioresistance, but was also accompanied by more aggressive invasion and migration abilities. Our previous studies discovered that the WNT signaling pathway was increasingly activated in radioresistance NPC cells $(6,12)$. Reversely, following WNT2B knockout, the NPC radioresistance and their invasion and migration capacities weakened (data not shown), along with corresponding changes in EMT markers (12). All the above findings suggest that WNT-mediated EMT may be a potential mechanism in NPC malignancy and warrants further investigation.

Chemotherapy is an indispensable method for the treatment of NPC, and our research firstly investigated the chemosensitivity of post-irradiation residual NPC cells. As first-line chemotherapeutics used in NPC treatment, paclitaxel and cisplatin were used in our study and multidrug resistance to both chemotherapeutic agents was observed in the radioresistant cells. Irradiation-induced multidrug resistance or an increase in related genes were also reported in other tumors, such as breast (25), oral (26) and colon (27). Even a low irradiation dose also led to chemoresistance $(28,29)$, suggesting that insufficient irradiation dose and residues may cause present or subsequent chemotherapy failure. In recent years, the role of EMT in drug resistance has attracted attention. In NPC, EMT is necessary for acquired resistance to cisplatin (30). The altered expression of FOXC2 and P53 could also affect NPC chemoresistance via regulation of EMT $(31,32)$.

The cancer stem cell (CSC) is considered as another crucial reason for advanced tumor malignancy. Via self-renewal and multi-direction differentiation, CSCs can survive in extreme situations such as exposure to irradiation or chemo-agents. The related mechanisms attributed to the CSC-mediated survival include high DNA damage repair capacity, cell cycle regulation and enhanced reactive oxygen species (ROS) defenses $(8,11)$. In our study, CSC-related genes Lgr5 and c-Myc were significantly upregulated in both radioresistance cell lines. In addition, higher proportions of Lgr5+ cells were observed in radioresistant cells. Lgr5 is a classic CSC biomarker and is associated with WNT pathway activation in many malignancies (33). c-Myc is reported as a key transcription factor for CSC phenotype maintenance $(34,35)$. Furthermore, during the therapy process, CSC trait transition and self-renewal could also elicit tumor adaptive responses by irradiation and the drug agents themselves (36).

Our present results indicate that co-existence of EMT and CSCs may be the common mechanisms for NPC radioresistance, chemotherapy tolerance, invasion and metastasis. In fact, the EMT phenotype may be the result of CSC multi-directional differentiation and may be a bridge to connect CSCs and treatment resistance (37). Thus, reversing the progression of EMT and CSCs may become a method to restrict multiple malignant bio-behaviors of NPC cells, and become an effective strategy to improve the prognosis and life quality of NPC patients with a residual and recurrent mass. These findings warrant further study.

\section{Acknowledgements}

Grants were provided by the National Natural Science Foundation of China (nos. 81372426, 81202128 and 81172558), the National Natural Science Foundation of Hunan Province (nos. 2015JJ3137 and 14JJ2018), and the Research Fund for the Doctoral Program of Higher Education of China (no. 20120162120049).

\section{References}

1. Yu MC and Yuan JM: Epidemiology of nasopharyngeal carcinoma. Semin Cancer Biol 12: 421-429, 2002.

2. Caponigro F, Longo F, Ionna F and Perri F: Treatment approaches to nasopharyngeal carcinoma: A review. Anticancer Drugs 21: 471-477, 2010.

3. Huang WY, Lin CL, Lin CY, Jen YM, Lo CH, Sung FC and Kao CH: Survival outcome of patients with nasopharyngeal carcinoma: A nationwide analysis of 13407 patients in Taiwan. Clin Otolaryngol 40: 327-334, 2015.

4. Li JX, Huang SM, Jiang XH, Ouyang B, Han F, Liu S, Wen BX and $\mathrm{Lu}$ TX: Local failure patterns for patients with nasopharyngeal carcinoma after intensity-modulated radiotherapy. Radiat Oncol 9: 87, 2014.

5. Li G, Qiu Y, Su Z, Ren S, Liu C, Tian Y and Liu Y: Genome-wide analyses of radioresistance-associated miRNA expression profile in nasopharyngeal carcinoma using next generation deep sequencing. PLoS One 8: e84486, 2013.

6. Li G, Liu Y, Su Z, Ren S, Zhu G, Tian Y and Qiu Y: MicroRNA-324-3p regulates nasopharyngeal carcinoma radioresistance by directly targeting WNT2B. Eur J Cancer 49: 2596-2607, 2013.

7. Yang $\mathrm{J}$ and Weinberg RA: Epithelial-mesenchymal transition: At the crossroads of development and tumor metastasis. Dev Cell 14: 818-829, 2008. 
8. Marie-Egyptienne DT, Lohse I and Hill RP: Cancer stem cells, the epithelial to mesenchymal transition (EMT) and radioresistance: Potential role of hypoxia. Cancer Lett 341: 63-72, 2013.

9. Kajiyama H, Shibata K, Terauchi M, Yamashita M, Ino K, Nawa A and Kikkawa F: Chemoresistance to paclitaxel induces epithelial-mesenchymal transition and enhances metastatic potential for epithelial ovarian carcinoma cells. Int J Oncol 31: 277-283, 2007.

10. Rycaj K and Tang DG: Cancer stem cells and radioresistance. Int J Radiat Biol 90: 615-621, 2014.

11. Di C and Zhao Y: Multiple drug resistance due to resistance to stem cells and stem cell treatment progress in cancer (review). Exp Ther Med 9: 289-293, 2015.

12. Li G, Wang Y, Liu Y, Su Z, Liu C, Ren S, Deng T, Huang D, Tian $Y$ and Qiu Y: miR-185-3p regulates nasopharyngeal carcinoma radioresistance by targeting WNT2B in vitro. Cancer Sci 105: 1560-1568, 2014

13. Yu C, Liu Y, Tan H, Li G, Su Z, Ren S, Zhu G, Tian Y, Qiu Y and Zhang X: Metadherin regulates metastasis of squamous cell carcinoma of the head and neck via AKT signalling pathway-mediated epithelial-mesenchymal transition. Cancer Lett 343: 258-267, 2014.

14. Schmittgen TD and Livak KJ: Analyzing real-time PCR data by the comparative C(T) method. Nat Protoc 3: 1101-1108, 2008.

15. Kaufhold S and Bonavida B: Central role of Snaill in the regulation of EMT and resistance in cancer: A target for therapeutic intervention. J Exp Clin Cancer Res 33: 62, 2014.

16. Spillane JB and Henderson MA: Cancer stem cells: A review. ANZ J Surg 77: 464-468, 2007.

17. Li WF, Zhang L, Li HY, Zheng SS and Zhao L: WISP-1 contributes to fractionated irradiation-induced radioresistance in esophageal carcinoma cell lines and mice. PLoS One 9: e94751, 2014.

18. Feng XP, Yi H, Li MY, Li XH, Yi B, Zhang PF, Li C, Peng F, Tang CE, Li JL, et al: Identification of biomarkers for predicting nasopharyngeal carcinoma response to radiotherapy by proteomics. Cancer Res 70: 3450-3462, 2010.

19. Zhang X, Zheng L, Sun Y, Wang T and Wang B: Tangeretin enhances radiosensitivity and inhibits the radiation-induced epithelial-mesenchymal transition of gastric cancer cells. Oncol Rep 34: 302-310, 2015.

20. Ni J, Cozzi PJ, Hao JL, Beretov J, Chang L, Duan W, Shigdar S, Delprado WJ, Graham PH, Bucci J, et al: CD44 variant 6 is associated with prostate cancer metastasis and chemo-/radioresistance. Prostate 74: 602-617, 2014.

21. de Jong MC, Ten Hoeve JJ, Grénman R, Wessels LF, Kerkhoven R, Te Riele H, van den Brekel MW, Verheij M and Begg AC: Pretreatment microRNA expression impacting on epithelial-to-mesenchymal transition predicts intrinsic radiosensitivity in head and neck cancer cell lines and patients. Clin Cancer Res 21: 5630-5638, 2015.

22. Cojoc M, Peitzsch C, Kurth I, Trautmann F, Kunz-Schughart LA, Telegeev GD, Stakhovsky EA, Walker JR, Simin K, Lyle S, et al Aldehyde dehydrogenase is regulated by $\beta$-catenin/TCF and promotes radioresistance in prostate cancer progenitor cells. Cancer Res 75: 1482-1494, 2015.

23. Chang L, Graham PH, Hao J, Ni J, Bucci J, Cozzi PJ, Kearsley JH and $\mathrm{Li}$ Y: Acquisition of epithelial-mesenchymal transition and cancer stem cell phenotypes is associated with activation of the $\mathrm{PI} 3 \mathrm{~K} / \mathrm{Akt} / \mathrm{mTOR}$ pathway in prostate cancer radioresistance. Cell Death Dis 4: e875, 2013.
24. Yan S, Wang Y, Yang Q, Li X, Kong X, Zhang N, Yuan C, Yang N and Kong B: Low-dose radiation-induced epithelial-mesenchymal transition through NF- $\mathrm{BB}$ in cervical cancer cells. Int J Oncol 42: 1801-1806, 2013.

25. Bottke D, Koychev D, Busse A, Heufelder K, Wiegel T, Thiel E, Hinkelbein W and Keilholz U: Fractionated irradiation can induce functionally relevant multidrug resistance gene and protein expression in human tumor cell lines. Radiat Res 170: 41-48, 2008.

26. Ng IO, Lam KY, Ng M, Kwong DL and Sham JS: Expression of P-glycoprotein, a multidrug-resistance gene product, is induced by radiotherapy in patients with oral squamous cell carcinoma. Cancer 83: 851-857, 1998

27. Bartkowiak D, Stempfhuber M, Wiegel T and Bottke D: Radiation- and chemoinduced multidrug resistance in colon carcinoma cells. Strahlenther Onkol 185: 815-820, 2009.

28. Eichholtz-Wirth $\mathrm{H}$ and Hietel B: Cisplatin resistance in mouse fibrosarcoma cells after low-dose irradiation in vitro and in vivo. Br J Cancer 70: 579-584, 1994

29. Hill BT, Moran E, Etiévant C, Perrin D, Masterson A, Larkin A and Whelan RD: Low-dose twice-daily fractionated X-irradiation of ovarian tumor cells in vitro generates drug-resistant cells overexpressing two multidrug resistance-associated proteins, P-glycoprotein and MRP1. Anticancer Drugs 11: 193-200, 2000.

30. Zhang P, Liu H, Xia F, Zhang QW, Zhang YY, Zhao Q, Chao ZH, Jiang ZW and Jiang CC: Epithelial-mesenchymal transition is necessary for acquired resistance to cisplatin and increases the metastatic potential of nasopharyngeal carcinoma cells. Int J Mol Med 33: 151-159, 2014.

31. Zhou Z, Zhang L, Xie B, Wang X, Yang X, Ding N, Zhang J, Liu Q, Tan G, Feng D, et al: FOXC2 promotes chemoresistance in nasopharyngeal carcinomas via induction of epithelial mesenchymal transition. Cancer Lett 363: 137-145, 2015.

32. Shen YA, Lin CH, Chi WH, Wang CY, Hsieh YT, Wei YH and Chen YJ: Resveratrol impedes the stemness, epithelial-mesenchymal transition, and metabolic reprogramming of cancer stem cells in nasopharyngeal carcinoma through p53 activation. Evid Based Complement Alternat Med 2013: 590393, 2013.

33. Yang L, Tang H, Kong Y, Xie X, Chen J, Song C, Liu X, Ye F, Li N, Wang N, et al: LGR5 promotes breast cancer progression and maintains stem-like cells through activation of Wnt/ $\beta$-catenin signaling. Stem Cells 33: 2913-2924, 2015.

34. Civenni G, Malek A, Albino D, Garcia-Escudero R, Napoli S, Di Marco S, Pinton S, Sarti M, Carbone GM and Catapano CV: RNAi-mediated silencing of Myc transcription inhibits stem-like cell maintenance and tumorigenicity in prostate cancer. Cancer Res 73: 6816-6827, 2013.

35. Moumen M, Chiche A, Decraene C, Petit V, Gandarillas A, Deugnier MA, Glukhova MA and Faraldo MM: Myc is required for $\beta$-catenin-mediated mammary stem cell amplification and tumorigenesis. Mol Cancer 12: 132, 2013.

36. Jachetti E, Mazzoleni S, Grioni M, Ricupito A, Brambillasca C, Generoso L, Calcinotto A, Freschi M, Mondino A, Galli R, et al: Prostate cancer stem cells are targets of both innate and adaptive immunity and elicit tumor-specific immune responses. OncoImmunology 2: e24520, 2013.

37. Dave B, Mittal V, Tan NM and Chang JC: Epithelial-mesenchymal transition, cancer stem cells and treatment resistance. Breast Cancer Res 14: 202, 2012. 